\title{
MINOR COMPOSITION COMPOUNDS OF ALGERIAN HERBAL MEDICINES AS INHIBITORS OF SARS-CoV-2 MAIN PROTEASE: MOLECULAR DOCKING AND ADMET PROPERTIES PREDICTION
}

\author{
B. YABRIR ${ }^{a}$, A. BELHASSAN ${ }^{b}$, T. LAKHLIFI $^{b}$, G. SALGADO M. $^{c}$, M. BOUACHRINE ${ }^{b}$, P. MUNOZ C. ${ }^{d}$, \\ L. GERLI C. ${ }^{e}$ AND R. RAMIREZ T. ${ }^{f}$
}

\author{
${ }^{a}$ Department of Biology, Faculty of Nature and Life Sciences, University Ziane Achour, Djelfa, Algeria. \\ ${ }^{b}$ Molecular Chemistry and Natural Substances Laboratory, Faculty of Science, Moulay Ismail University of Meknes, Morocco. \\ ${ }^{c}$ Facultad de Ciencias Quimicas. Investigador Extramural, Universidad de Concepcion, Concepcion, Chile. \\ ${ }^{d}$ Departamento de Ciencias Químicas, Facultad de Ciencias Exactas, Universidad Andrés Bello, Santiago, Chile. \\ ${ }^{e}$ Departamento de Química Ambiental, Facultad de Ciencias, Universidad Católica de la Santísima Concepción, Concepción, Chile. \\ ${ }^{f}$ Universidad Bernardo O'hhiggins, Laboratorio de Nanotechnologia, Santiago, Chile.
}

\begin{abstract}
The identification of drugs against the new coronavirus (SARS-CoV-2) is an important requirement. Natural products are substances that serve as sources of beneficial chemical molecules for the development of effective therapies. In this study, 187 natural compounds from Algerian herbal medicines were docked in the active site of SARS-CoV-2 main protease. The result indicates that Piperitol, Warfarin, cis-calamenen-10-ol and $\alpha$-Cadinene are the structures with best affinity in the binding site of the studied enzyme and all of them respect the conditions mentioned in Lipinski's rule and have acceptable ADMET proprieties; so, these compounds could have more potent antiviral treatment of COVID-19 than the studied compounds, and they have important pharmacokinetic properties and bioavailability.
\end{abstract}

Keywords: COVID-19, SARS-CoV-2, Algerian herbal, Natural compounds, Piperitol, ADMET, Molecular Docking.

\section{INTRODUCTION}

Emerging or re-emerging epidemic diseases pose a continuing threat to global health security, including Severe Acute Respiratory Syndrome CoronaVirus (SARS-CoV) [1], Middle East Respiratory Syndrome (MERS-CoV) [2], and recently a contagious respiratory illnesses called COronaVirus Infectious Disease 2019 (COVID-19) [3]. This later, highly publicized, is the most dangerous, which declared pandemic by WHO in March 2020 [4]. It is caused by infection with a new coronavirus, known as the severe acute respiratory syndrome coronavirus 2 (SARS-CoV-2), identified firstly in December 2019 in Wuhan (China) [5]. This novel beta-coronavirus is a positive-sense single stranded RNA virus with a genome of about $30 \mathrm{~kb}$ with structured untranslated region; belonging to the family coronaviruses and subgenus sarbecovirus (betaCoV lineage B), but it differs from previously known SARS-CoV and MERS$\mathrm{CoV}$ [6]. It bears about $80 \%$ genome homology with SARS-CoV and has about $96 \%$ identical genes with the bat coronavirus [3]. Symptom of COVID-19 are non-specific and the disease presentation can range from no symptoms (asymptomatic) to severe pneumonia and death [7] which fever, cough, shortness of breath, chills, muscle pain, new loss of taste or smell, vomiting or diarrhea, and/or sore throat are common symptoms seen in adults [8]. Among others, and according to Siordia Jr. The most common comorbidity is hypertension (30.7\%), followed by diabetes mellitus (14.3\%) and cardiovascular diseases (11.9\%) [9].

So far, all researchers agree that there is no specific treatment for COVID-19. So, without any effective antiviral agents and no approved human coronavirus vaccines available at present, and to prevent respiratory aerosol/droplet infection generated by individuals who are symptomatic, and further spread of COVID19, some precautions are recommend for avoiding COVID-19, as isolation of the patient, hand hygiene measures, use of face masks and other sanitary measures. This will reduce the risk of transmission to care givers and close contacts $[3,10]$. Current treatments focus on managing symptoms along the course of infection [11]. Meanwhile, numerous research laboratories are currently seeking a treatment that can eliminate the MERS-CoV infection, by searching for new more specific alternatives for the virus [12]. A need for effective vaccine is being seen an as good preventive strategy in this pandemic [3]. So, a variety of antiviral agents and symptomatic treatments are being administered to patients and urgent clinical trials are underway, thus under these circumstances, it is important to explore various possibilities for the treatment of COVID-19 including herbal medicines [13]. Clarification transmission routes and pathogenic mechanisms, and identification of potential drug treatment targets will promote the development of effective prevention and treatment measures [14].

Basis on its historical experience and holistic pharmacological action, traditional Chinese medicine (TCM) could be used as an alternative treatment option or in combination with Western medicine to treat COVID-19 [15]. TCM was used in $91.50 \%$ of the COVID-19 cases in China, showing encouraging results in improving symptom management and reducing the deterioration, mortality, and recurrence rates [16]. Many medicinal herbs were used in TCM formulae for treating COVID-19 in China [17,18], some of them has been prescribed to COVID-19 patients according to the status of their disease (mild, moderate, severe, and critical stages) $[11,17]$. Panyod et al. summarize the antiviral activity of foods and herbs against influenza virus [19], and results of ongoing clinical trials on hydroxychloroquine, azithromycin alone or in combination and a new antiviral agent remdesivir may help to treat some of the infections [3]. Unfortunately, the two first drugs have toxic effects and their use in high doses can create major risks for patients [20,21].

Based on in vitro and in vivo studies, promising compounds in traditional medicine can be used as effective antiviral drugs for the treatment of diseases caused by SARS-CoV-2 [14]. The anti-CoVs effects of natural compounds and their possible action mechanisms are reported in the literature review of Xian et al. [22]. These molecules can act as inhibitors of replication, membrane fusion and assembly of SARS-CoV-2 [23]. Additionally, computer molecular docking shows that these monomers have good binding ability to COVID-19 virus and host targets [14]. Despite, promising results obtained from molecular docking of selected drugs with potential targets do not guarantee the effectiveness of a drug in treatment under clinical conditions; however, these works provide a general idea of where research should go [24].

In this paper, 187 natural compounds were docked into the active site of SARS$\mathrm{CoV}-2$ main protease to predict the mode of binding between these molecules and their potential target (SARS-CoV-2 main protease), also to determine the affinity of these molecules in the binding site of the studied enzyme. The study is followed by a prediction of Lipinski's rule and ADMET parameters of the studied compounds, chloroquine and hydroxychloroquine; the aim of which is to find substances more effective than chloroquine and hydroxychloroquine which do not have toxic effects of these two drugs. 


\section{MATERIALS AND METHODS}

\subsection{Data Collection}

This article constitutes the continuation of our previous work about major components of medicinal plants collected from Algerian pharmacopeia which were docked in the active site of SARS-CoV-2 main protease as possible inhibitors. unlike major compounds, the minor's ones are generally neglected in scientific research. These compounds may elicit some activity, while in pure form, against organisms although the proportions of such compounds in the plant were considerably low; on the other hand, minor components are critical to the activity and may have a synergistic effect or potentiating influence.

Species chosen are those found in bibliographic data and which are used in Algerian traditional medicine in the treatment of diseases of the respiratory

Table 1. Chemical composition of medicinal plants and their percentages. system including cough, Rhinitis, Bronchitis, Asthma, Influenza, Flu and Pulmonary diseases in general, according traditional Algerian pharmacopeia

In this work, we have selected 187 compounds extracted from 24 different aromatic and medicinal plants from Algeria; Table 1 shows information on the plant of origin of each compound used as well as their percentages in the plant. The selection is based on these criteria:

- Traditional medicinal use: based on infections and diseases related to the respiratory system;

- The chemical constituents are those declared minority by the authors;

- All items declared correspond to medicinal species collected in Algeria.

\begin{tabular}{|c|c|c|c|c|c|c|c|c|c|}
\hline \multirow{2}{*}{$\mathbf{N}^{\circ}$} & \multirow{2}{*}{ Minor constituents (\%) } & \multirow{2}{*}{ Scientific/ (Common) Name } & \multicolumn{5}{|c|}{ Therapeutic use ${ }^{*}$} & \multirow{2}{*}{ Used parts } & \multirow{2}{*}{ Ref. } \\
\hline & & & 1 & 2 & 3 & 4 & 5 & & \\
\hline 1 & $\begin{array}{l}\alpha \text {-Thujone (0.6), Aldehydeperrilique (0.6), } \beta \text {-Caryophyllene (0.6), Alcool perrilique (0.5), 3,4- } \\
\text { Dimethyl cinnoline (0.4), nor } \beta \text {-Calamenene }(0.4), \alpha \text {-Copaene }(0.1)\end{array}$ & $\begin{array}{c}\text { Artemisia arborescens } L . \\
\text { (Sayba, siba, sejert meriem) }\end{array}$ & $\checkmark$ & & & & & $\begin{array}{c}\text { Aerial part, } \\
\text { leaves }\end{array}$ & {$[25]$} \\
\hline 2 & $\begin{array}{l}\text { Limonene }(0.3), 6,8 \text {-Nonadien-2-one,6-methyl-5-(1-methyletildene) }(0.3), \gamma \text {-Muurolene }(0.3), \\
\alpha \text {-Zingiberene(0.3), Methyl p-tert-buthylphenil acetate }(0.3), 3 \text {-Otadecine }(0.3)\end{array}$ & $\begin{array}{c}\text { Peganum harmala } \mathrm{L} . \\
\text { (Harmel) }\end{array}$ & $\checkmark$ & & & & & $\begin{array}{c}\text { Aerial part, } \\
\text { seeds }\end{array}$ & {$[26]$} \\
\hline 3 & $\begin{array}{l}\text { (E)-Salvene (0.1), cis-Verbenol }(0.1) \text {, Borneol }(0.1) \text {, Thymol }(0.1), Z \text { - } \beta \text {-Damascenone }(0.1) \text {, } \\
\text { Aromadendrene }(0.1), \delta \text {-Cadinene }(0.1) \text {, Spathulenol(0.1), Caryophyllene oxide }(0.1)\end{array}$ & $\begin{array}{l}\text { Pinus halepensis Mill. } \\
\text { (Sanouber halabi) }\end{array}$ & $\checkmark$ & & $\checkmark$ & $\checkmark$ & & $\begin{array}{c}\text { Resin, fruits, } \\
\text { leaves and } \\
\text { tree bark }\end{array}$ & {$[27]$} \\
\hline 4 & $\begin{array}{l}\text { (z)-Tetradec-9-enoic acid (0.3), (E)-Tetradec-9-enoic acid }(0.3) \text {, Methylhexadecanoate } \\
(0.2) \text {,Ethylheptadecanoate }(0.3) \text {, Ethyloctradecanoate }(0.3 \%) \text {, Tricosane }(0.3) \text {, Tetracosane }(0.3), \\
\text { Hexacos-9-ene }(0.2) \text {, Hexacosane }(0.3)\end{array}$ & Ziziphus lotus L. (Sedra) & $\checkmark$ & & & & & $\begin{array}{l}\text { Leaves, } \\
\text { Roots }\end{array}$ & {$[28]$} \\
\hline 5 & $\begin{array}{l}\text { Benzene (0.3), Cyclohexane (0.3), 2, 3- Butanediol(0.4), cis-2-Hexenal(0.3), 3-Hexen-1-ol (0.3), } \\
\text { 1-Hexanol }(0.5 \%) \text {, Nonanal (0.5) }\end{array}$ & $\begin{array}{l}\text { Marrubium deserti de Noé } \\
\text { (Temiriouet er'rmel) }\end{array}$ & $\checkmark$ & $\checkmark$ & $\checkmark$ & $\checkmark$ & $\checkmark$ & Whole plant & [29] \\
\hline 6 & $\begin{array}{l}\text { Camphene (0.1), } \beta \text {-Pinene (0.2-0.4),Myrcene (0.1-0.4), y-Terpinene (0.2-0.3), Carvenone (0.2), } \\
\text { Pinocarvone(O.1-0.3), Aromadendrene (0,2-0,6), Terpinen-4-01 (0,3-0,5), Allo-aromadendrene } \\
(0.1-0.5), \alpha \text {-Terpineol }(0.1), \delta \text {-Terpineo (0.1), p-Mentha-1(7),8-dien-2-ol (0.2-0.3), p-Cymen-8- } \\
01(0.4), \text { trans-Carveol (0.1), Piperitol (0.2), epi-Globulol (0.2-0.4), Globulol (0.2-0.6), } \alpha- \\
\text { Eudesmol (0.1-0.2) }\end{array}$ & $\begin{array}{r}\text { Eucalyptus sp. } \\
\text { (Kalitousse) }\end{array}$ & $\checkmark$ & & & & & Leaves & {$[30]$} \\
\hline 7 & $\begin{array}{l}\text { Sabinene }(0.2-0.7) 2 \text {-Phenylethyl alcohol }(0.2) \text {, Indole }(0.3), \alpha \text {-Copaene }(0.2), \beta \text {-Bourbonene }(0.1), \\
\beta \text {-Elemene }(0.2), \alpha \text {-Humulene }(0.4) \text {, rans- } \beta \text {-Farnesene }(0.3), \beta \text {-Ionone }(0.1), \text { Germacrene D }(0.3), \\
\text { Pentadecane }(0.5), \alpha \text {-Cadinene }(0.3)\end{array}$ & $\begin{array}{l}\text { Marrubium vulgare L. } \\
\quad \text { (Temiriouet) }\end{array}$ & & & & & $\checkmark$ & Aerial part & {$[31]$} \\
\hline 8 & $\begin{array}{l}\alpha \text {-Thujene }(0.1) \text {, Camphene }(0.1) \text {, Linalool }(0.1 \%), \text { Borneol }(0.1), \alpha \text {-Cubebene }(0.1), \alpha \text {-Gurjunene } \\
(0.1) \text {, trans-Caryophyllene }(0.1), \beta \text {-Gurjunene }(0.1), \delta \text {-Cadinene }(0.1), \quad \text { Cadina-1,4-diene }(0.1), \\
\text { trans-Nerolidol }(0.1), \text { Abietatriene }(0.1)\end{array}$ & $\begin{array}{l}\text { Juniperus oxycedrus L. } \\
\text { (Arar, Taga) }\end{array}$ & $\checkmark$ & & $\checkmark$ & & & Aerial part & {$[32]$} \\
\hline 9 & $\begin{array}{l}\text { Limonene( } 0.6) \text {, Sabinène (0.4), -amorphène (0.4), Tridécanal }(0.5), \beta \text {-eudesmol }(0,5), \alpha \text {-eudesmol } \\
(0,4)\end{array}$ & $\begin{array}{l}\text { Pistacia atlantica Desf. } \\
\text { (Botom) }\end{array}$ & $\checkmark$ & & $\checkmark$ & & & Resin \& fruit & {$[33]$} \\
\hline 10 & $\begin{array}{l}\text { Hexanal(0.09), Octan-3-ol (0.05), 6-Methyl-hepta-3,5-dien-2-one(0.09), Terpinene-4-ol (0.07), } \\
\text { Elemol (0.08), Megastigmatrienone2 (0.09), Ethyldibenzenothiophene (0.09) }\end{array}$ & $\begin{array}{c}\text { Genista saharae } \\
\text { (Tellegit) }\end{array}$ & $\checkmark$ & $\checkmark$ & $\checkmark$ & $\checkmark$ & $\checkmark$ & Arial parts & {$[34]$} \\
\hline 11 & 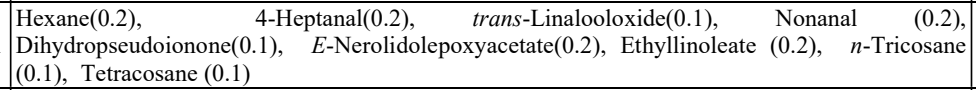 & $\begin{array}{l}\text { Matricaria pubescens } \\
\text { (Guertoufa) }\end{array}$ & $\checkmark$ & & & $\checkmark$ & & Arial parts & {$[35]$} \\
\hline 12 & $\begin{array}{l}\text { Camphene (0.20), } \beta \text {-Pinene (0.37), 3-Carene (0.36), Myrcene (0.11), Methyl perillate (0.92), } \\
\text { Perilla alcohol (0.67), Spathulenol (0.35) }\end{array}$ & $\begin{array}{l}\text { Ammodaucus leucotrichus } \\
\text { (El-Kammoun essofi, el } \\
\text { massoufa) }\end{array}$ & $\checkmark$ & & & & & $\begin{array}{l}\text { Fruits, seed, } \\
\text { leaves }\end{array}$ & {$[36]$} \\
\hline 13 & $\begin{array}{|lcccc|}\begin{array}{l}\text { Terpinene }(0.22), \\
\text { Spathlenol(0.10), Globulol }(0.19), \text { Farnesol }(0.30)\end{array} & \beta \text {-Guaiene }(0.20), & \text { Trans-calamenene }(0.12), \\
\end{array}$ & $\begin{array}{c}\begin{array}{c}\text { Ephedra alata } \\
\text { (Alanda) }\end{array} \\
\end{array}$ & $\checkmark$ & & $\checkmark$ & $\checkmark$ & $\checkmark$ & Arial parts & {$[37]$} \\
\hline 14 & 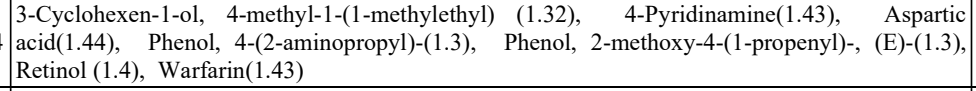 & $\begin{array}{l}\text { Glycyrrhiza glabra } \\
\text { (Erg essous) }\end{array}$ & $\checkmark$ & & & & & Roots & {$[38]$} \\
\hline 15 & \begin{tabular}{|l|}
$\alpha$-Thujene $(0.10-1.12)$, Sabinene $(0.10), \beta$-Myrcene $(0.18-0.22), \alpha$-Terpinene $(0.15-0.16)$, \\
Methylcitronellate (0.11-0.68), Exo-2-hydroxycineole acetate $(0.17-0.21),($ E)-Methyl isoeugenol \\
$(0.11-0.17), \gamma$-Elemene (0.10), Spathulenol (0.10-0.12), Cubenol (0.18), $\beta$-Eudesmol $(0,15-$ \\
$024 \%)$
\end{tabular} & \begin{tabular}{|c|} 
Myrtus communis \\
(Al-Rihan or el-halmouche)
\end{tabular} & $\checkmark$ & $\checkmark$ & $\checkmark$ & $\checkmark$ & $\checkmark$ & $\begin{array}{l}\text { Leaves, } \\
\text { buds, fruits }\end{array}$ & [39] \\
\hline 16 & $\begin{array}{l}\alpha \text {-Thujene }(0.15) \text {, Thuja-2,4(10)-diene }(0.10), \alpha \text {-Terpinene }(0.15) \text {, Terpinolene }(0.07) \text {, Nopinone } \\
(0.10) \text {, Sabina ketone }(0.14) \text {, Carvacrol acetate }(0.6), \alpha \text {-elemene }(0.03), \beta \text {-Copaene }(0.12), \alpha \text { - } \\
\text { Guaiene }(0.15) \text {, trans- } \beta \text {-Elemenone }(0.15)\end{array}$ & $\begin{array}{l}\text { Thymus ciliatus (Desf.) } \\
\text { (Zaatar) }\end{array}$ & $\checkmark$ & & $\checkmark$ & & & Arial parts & {$[40]$} \\
\hline 17 & $\begin{array}{l}\alpha \text {-Thujene }(0.05), \alpha \text {-Phellandrene }(0.01), \delta \text {-Carene }(0.01),(Z)-\beta \text {-Ocimene }(0.03) \text {, allo-Ocimène } \\
(0.02) \text {, Thymol }(0.03), \text { Carvacrol }(0.02), \text { allo-Aromadendrene }(0.07), \delta \text {-Cadinene }(0.08),(Z)- \\
\text { Nerolidol }(0.04)\end{array}$ & $\begin{array}{l}\text { Thymus algeriensis } \\
\quad \text { (Zaatar) }\end{array}$ & $\checkmark$ & $\checkmark$ & $\checkmark$ & $\checkmark$ & $\checkmark$ & $\begin{array}{l}\text { Summits, } \\
\text { young } \\
\text { flowering } \\
\text { twigs }\end{array}$ & \\
\hline 18 & $\begin{array}{l}\text { Sabinene }(0.04), \beta \text {-Pinene }(0.19), \alpha \text {-Phellandrene }(0.19), \delta \text {-Carene }(0.07), \quad \text { Limonene }(0.17), \\
\text { Terpinolene }(0.16), \alpha \text {-Thujone }(0.07), \quad \text { Camphor }(0.03), \quad \text { Naphtalene }(0.08), \quad \text { Aromadendrene } \\
(0.10) \text {, allo-Aromadendrene(0.05), Germacrene D }(0.03)\end{array}$ & $\begin{array}{l}\text { Thymus vulgaris } \\
\text { (Zaatar) }\end{array}$ & & & $\checkmark$ & & $\checkmark$ & Arial parts & {$[41]$} \\
\hline 19 & $\begin{array}{l}\alpha \text {-Thujene }(0.05), \alpha \text {-Pinene }(0.06) \text {, Camphene }(0.02) \text {, Sabinene }(0.09), \alpha \text {-Phellandrene }(0.01), \delta \text { - } \\
\text { Carene }(0.04) \text {, allo-Ocimène }(0.05) \text {, Isopulegol }(0.10) \text {, Naphtalene }(0.11)\end{array}$ & $\begin{array}{l}\text { Mentha pulegium } \\
\text { (Fleyou) }\end{array}$ & $\checkmark$ & & $\checkmark$ & & & $\begin{array}{c}\text { flowering } \\
\text { aerial } \\
\text { parts }\end{array}$ & \\
\hline 20 & $\begin{array}{l}\text { Octane -3-one ( } 0.03) \text {, Borneol }(0.02) \text {, Thymoquinone }(0.04) \text {, Eugenol (0.04), Trans-carvyl acetate } \\
(0.02) \text {, Ylangene (0.03), Cis-jasmone }(0.04), \alpha \text {-muurolene }(0.04) \text {, Cadina1-4-diene }(0.03), \alpha \text { - } \\
\text { cadinene }(0.05) \text {, Viridiflorol }(0.01) \text {, Ledol }(0.01), \beta \text {-eudesmol }(0,01)\end{array}$ & $\begin{array}{l}\text { Thymus numidicus Poiret } \\
\text { (Zaatar) }\end{array}$ & $\checkmark$ & $\checkmark$ & $\checkmark$ & $\checkmark$ & $\checkmark$ & Arial parts & {$[42]$} \\
\hline
\end{tabular}




\begin{tabular}{|c|c|c|c|c|c|c|c|c|}
\hline 21 & $\begin{array}{l}\text { p-Cymene }(0.23), \gamma \text {-Terpinene }(0.21), 1 \text {-Octen-3 ylacetate }(0.24), 3 \text {-Octanyl acetate }(0.26), \\
\text { Carvone }(0.16), \text { Lavandulylacetate }(0.23) \text {, Myrtenylacetate }(0.1), B \text {-Elemene }(0.26), \alpha \text {-Gurjunene } \\
(0.1) \text {, Germacrene D }(0.2), \delta \text {-Cadinene }(0.26), \alpha \text {-Eudesmol }(0,20)\end{array}$ & $\begin{array}{l}\text { Ocimum basilicum } \\
\text { (Hbek) }\end{array}$ & $\checkmark$ & & & & $\begin{array}{c}\text { Aerial parts, } \\
\text { leaves }\end{array}$ & [43] \\
\hline 22 & $\begin{array}{l}\text { Neo Allo-Ocimene (0.2), } \alpha \text {-Terpineol }(0.1), \quad \mathrm{n} \text { Octyl acetate }(0.2), \alpha \text {-Bergamotene }(0.1), \\
\text { Bicyclogermacrene }(0.2) \text {, Germacrene D (0.2), } \alpha \text {-Amorphene (0.2), Spathulenol }(0.2), \gamma \text {-Eudesmol } \\
(0,2), \beta \text {-Eudesmol }(0,1), \alpha \text {-Eudesmol }(0,1)\end{array}$ & $\begin{array}{c}\text { Ocimum gratissimum } \\
\text { (Hbek) }\end{array}$ & $\checkmark$ & & & & $\begin{array}{c}\text { Aerial parts, } \\
\text { leaves }\end{array}$ & [44] \\
\hline 23 & $\begin{array}{l}\text { 1-octen-3-ol }(0.1), \alpha \text {-terpinene }(0.1),(Z) \text { - } \beta \text {-ocimene }(0.1) \text {, benzene acetaldehyde }(0.1), 1 \text {-nonen-3- } \\
\text { ol }(0.1), p \text {-mentha-2,4(8)-diene }(0.1), \text { myrtenol }(0.1), \text { trans-dihydrocarvone }(0.1), \text { verbenone } \\
(0.1), \text { carvacrol, methyl ether }(0.1), \text { indole }(0.1), \beta \text {-cubebene }(0.1), \beta \text {-elemene }(0.1), \beta \text {-copaene } \\
(0.1), \gamma \text {-himachalene }(0.1), \beta \text {-bisabolene }(0.1), \beta \text {-sesquiphellandrene }(0.1), 1 \text {-nor-bourbonanone } \\
(0.1), \text { viridiflorol }(0.1), \text { salvial-4(14)-en-1-one }(0.1), 1,10 \text {-di-epi-cubenol }(0.1), \text { epi- } \alpha \text {-muurolol } \\
(0.1), \text { cis-calamenen-10-ol }(0.1), \alpha \text {-bisabolol }(0.1),\end{array}$ & $\begin{array}{l}\text { Thymus munbyanus } \\
\text { (Jertil et Ziitra) }\end{array}$ & $\checkmark$ & $\checkmark$ & & $\checkmark$ & $\begin{array}{l}\text { Leaves and } \\
\text { flowering } \\
\text { aerial parts }\end{array}$ & [45] \\
\hline 24 & 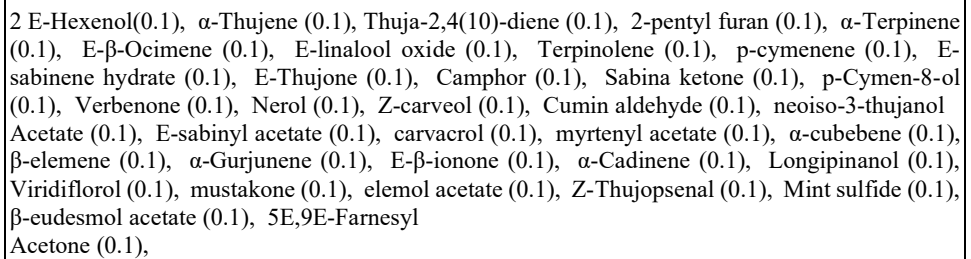 & $\begin{array}{c}\text { Teucrium polium } \\
\text { (Khayatta ou Djaâda) }\end{array}$ & & & $\checkmark$ & & $\begin{array}{l}\text { Aerial pars, } \\
\text { leaves }\end{array}$ & [46] \\
\hline
\end{tabular}

*1: cough; 2: Rhinitis; 3: Bronchitis; 4: Asthma; 5: Influenza, Flu; ${ }^{*}$ Some of characteristics and properties of medicinal plants are extracted from Quezel and Santa [47] and IUCN [48].

\subsection{Molecular Docking}

All studied compounds were obtained from chemical structure databases ChemSpider (An Online Chemical Information Resource) [49]. Molecular docking carried out to determine binding affinity and predict the intermolecular interactions of molecules in receptors. We performed a docking of studied compounds in the binding pocket of SARS-CoV-2 main protease (pdb code 6LU7) [50].

The docking study was carried out with two programs; Autodock vina [51] and Autodock tools 1.5.6 [52]. such programs are usually used to dock known substrates into the active site or known protein-binding molecules to the surface of the model [53]. The crystallographic structure of SARS-CoV-2 main protease (pdb code 6LU7) is imported into "work space" of Discovery Studio 2016 program [54] to obtain the binding site [55]. The center of the active site has been determined and it corresponds to the coordinates: $x=-10.782, y=15.787$ and $\mathrm{z}=71.277$ on the basis of the co-crystallized bound peptidomimetic ligand [56]. The grid size was set at $20 \times 20 \times 20$ xyz points with a grid spacing of $1 \AA$ to cover the folic acid binding site in the enzyme and was generated by using the cocrystallized ligand (N3) as the center for docking [56]. For ligand and enzyme preparations; an extended PDB format, termed PDBQT, is used for coordinate files, which includes atomic partial charges and atom types. Torsion angles were calculated to assign the flexible and non-bonded rotation of molecules. The results were subsequently analyzed using Discovery studio 2016 [54].

\subsection{Lipinski's Rule and ADMET Prediction}

The Lipinski's rule and ADMET [57] parameters (Absorption, Distribution, Metabolism, Excretion, Toxicity) of potential inhibitors were calculated using pkCSM [58] and Swissadmet [59] web servers. The Lipinski's rule including; molecular weight, $\log \mathrm{P}$, number of rotatable bonds, number of hydrogen bonds acceptor and number hydrogen bonds donor were determinate. Molecules violating more than one of these parameters may have problems with bioavailability and a high probability of failure to display drug-likeness [60].

\section{RESULTS AND DISCUSSIONS}

\subsection{Molecular Docking}

Molecular docking was performed to find types of interactions and the binding affinity of studied molecules in the studied enzyme. 187 different natural compounds have been evaluated for their affinity against the SARS-CoV-2 main protease (pdb code 6LU7). The results are presented in Table 2.

Table 2. Docking results: Affinity of the best conformation in the binding pocket of SARS-CoV-2 main protease.

\begin{tabular}{|c|c|c|c|c|c|c|c|c|}
\hline $\mathbf{N}^{\circ}$ & Name of molecules & $\begin{array}{c}\text { Affinity } \\
\text { (Kcal/mol) }\end{array}$ & $\mathbf{N}^{\circ}$ & Name of molecules & $\begin{array}{c}\text { Affinity } \\
\text { (Kcal/mol) }\end{array}$ & $\mathbf{N}^{\circ}$ & Name of molecules & $\begin{array}{c}\text { Affinity } \\
\text { (Kcal/mol) }\end{array}$ \\
\hline 1 & $\alpha$-Thujone & -4.9 & 64 & a-Cadinene & -6.1 & 127 & Camphore & -4.5 \\
\hline 2 & Perillaldehyde & -4.7 & 65 & $\alpha$-Thujene & -4.1 & 128 & Naphtalene & -4.8 \\
\hline 3 & $\beta$-caryophyllene & -5.8 & 66 & Linalool & -4.8 & 129 & $\alpha$-Pinene & -4.8 \\
\hline 4 & Perillyl alcohol & -4.8 & 67 & $\alpha$-Cubebene & -5.6 & 130 & Isopulegol & -4.5 \\
\hline 5 & 3,4-dimethylcinnoline & -5.5 & 68 & $\alpha$-Gurjunene & -5.3 & 131 & Octane-3-one & -3.9 \\
\hline 6 & $\alpha$-copaene & -5.1 & 69 & E-caryophyllene & -5.2 & 132 & Thymoquinone & -5.0 \\
\hline 7 & Limonene & -4.5 & 70 & $\beta$-Gurjunene & -5.5 & 133 & Eugenol & -4.9 \\
\hline 8 & $\begin{array}{c}\text { 6,8-Nonadien-2-one, } 6- \\
\text { methyl-5-(1- } \\
\text { methylethylidene)- }\end{array}$ & -5.0 & 71 & Cadina-1,4-diene & -5.6 & 134 & Trans-carvyl acetate & -5.1 \\
\hline 9 & $\gamma$-Muurolene & -5.3 & 72 & Trans-Nerolidol & -5.2 & 135 & Ylangene & -5.3 \\
\hline 10 & $\alpha$-Zingiberene & -5.6 & 73 & Abietatriene & -6.4 & 136 & Cis-jasmone & -4.8 \\
\hline 11 & $\begin{array}{l}\text { Methyl p-tert-butylphenyl } \\
\text { acetate }\end{array}$ & -5.5 & 74 & $\alpha$-amorphene & -5.3 & 137 & $\alpha$-Muurolene & -5.4 \\
\hline 12 & (E)-3-Octadecene & -4.2 & 75 & Tridecanal & -4.2 & 138 & Viridiflorol & -5.8 \\
\hline 13 & (E)-Salvene & -4.3 & 76 & $\beta$-Eudesmol & -5.7 & 139 & Ledol & -5.3 \\
\hline
\end{tabular}




\begin{tabular}{|c|c|c|c|c|c|c|c|c|}
\hline 14 & Cis-verbenol & -4.4 & 77 & Hexanal & -3.3 & 140 & p-Cymene & -4.6 \\
\hline 15 & Borneol & -4.3 & 78 & Octan-3-ol & -3.8 & 141 & 1-Octen-3-ylacetate & -4.0 \\
\hline 16 & Thymol & -4.7 & 79 & 6-Methyl-hepta-3,5-dien-2-one & -4.6 & 142 & (3R)-3-Octanyl acetate & -4.1 \\
\hline 17 & Z- $\beta$-Damascenone & -4.9 & 80 & Terpinene-4-ol & -4.7 & 143 & Carvone & -4.8 \\
\hline 18 & Aromadendrene & -5.3 & 81 & Elemol & -5.1 & 144 & Lavandulyl acetate & -4.9 \\
\hline 19 & $\delta$-cadinene & -5.8 & 82 & Megastigmatrienone & -5.3 & 145 & Myrtenyl acetate & -5.0 \\
\hline 20 & Spathulenol & -5.8 & 83 & Ethyldibenzothiophene & -5.7 & 146 & Neo-Allo-Ocimene & -4.7 \\
\hline 21 & Caryophyllene oxide & -5.5 & 84 & Hexane & -3.0 & 147 & n-Octyl acetate & -4.1 \\
\hline 22 & (z)-Tetradec-9-enoic acid & -4.4 & 85 & 4-Heptanal & -3.7 & 148 & $\alpha$-Bergamotene & -5.0 \\
\hline 23 & (E)-Tetradec-9-enoic acid & -5.0 & 86 & Trans-Linalool oxide & -5.0 & 149 & Bicyclogermacrene & -5.0 \\
\hline 24 & Methyl hexadecanoate & -4.3 & 87 & Dihydropseudoionone & -5.2 & 150 & $\gamma$-Eudesmol & -5.7 \\
\hline 25 & Ethylheptadecanoate & -5.0 & 88 & Ethyllinoleate & -4.6 & 151 & 1-Octen-3-ol & -3.8 \\
\hline 26 & Tricosane & -4.1 & 89 & n-Tricosane & -4.0 & 152 & Benzene acetaldehyde & -4.2 \\
\hline 27 & Tetracosane & -4.3 & 90 & 3-Carene & -4.5 & 153 & 1-nonen-3-ol & -4.1 \\
\hline 28 & Hexacos-9-ene & -4.5 & 91 & Methyl perillate & -4.9 & 154 & p-mentha-2,4(8)-diene & -5.0 \\
\hline 29 & Hexacosane & -4.5 & 92 & Perilla alcohol & -4.7 & 155 & Myrtenol & -5.0 \\
\hline 30 & Benzene & -3.4 & 93 & & & 156 & Trans-dihydrocarvone & -4.8 \\
\hline 31 & Cyclohexane & -3.2 & 94 & Bornyl acetate & -5.2 & 157 & Verbenone & -5.1 \\
\hline 32 & 2,3-Butanediol & -3.8 & 95 & $\beta$-Guaiene & -5.3 & 158 & Carvacrol methyl ether & -5.0 \\
\hline 33 & Cis-2-Hexenal & -3.6 & 96 & Trans-Calamenene & -5.9 & 159 & $\beta$-cubebene & -5.6 \\
\hline 34 & 3-Hexen-1-ol & -3.5 & 97 & Spathlenol & -5.6 & 160 & $\gamma$-himachalene & -5.4 \\
\hline 35 & 1-Hexanol & -3.5 & 98 & Farnesol & -5.4 & 161 & $\beta$-Bisabolene & -5.6 \\
\hline 36 & Nonanal & -3.9 & 99 & $\begin{array}{l}\text { 3-Cyclohexen-1-ol, 4-methyl- } \\
\text { 1-(1-methylethyl)-, acetate }\end{array}$ & -4.7 & 162 & $\beta$-sesquiphellandrene & -5.9 \\
\hline 37 & Camphene & -4.0 & 100 & 4-Pyridinamine & -3.7 & 163 & 1-nor-bourbonanone & -5.3 \\
\hline 38 & $\beta$-pinene & -4.6 & 101 & Aspartic acid & -4.7 & 164 & salvial-4(14)-en-1-one & -5.1 \\
\hline 39 & Myrcene & -4.0 & 102 & Phenol, 4-(2-aminopropyl)- & -4.8 & 165 & 1,10-di-epi-cubenol & -5.7 \\
\hline 40 & $\gamma$-terpinene & -4.6 & 103 & $\begin{array}{l}\text { Phenol, 2-methoxy-4-(1- } \\
\text { propenyl)-, (E)- }\end{array}$ & -5.2 & 166 & $\alpha$-epi-muurolol & -5.7 \\
\hline 41 & Carvenone & -4.8 & 104 & Retinol & -6.1 & 167 & cis-calamenen-10-ol & -6.3 \\
\hline 42 & Pinocarvone & -4.9 & 105 & Warfarin & -7.1 & 168 & $\alpha$-bisabolol & -5.8 \\
\hline 43 & Terpinen-4-ol & -4.3 & 106 & $\beta$-Myrcene & -4.8 & 169 & 2 E-Hexenol & -3.6 \\
\hline 44 & Allo-aromadendrene & -5.4 & 107 & $\alpha$-Terpinene & -4.6 & 170 & 2-pentyl furan & -4.1 \\
\hline 45 & $\alpha$-Terpineol & -4.8 & 108 & Methyl citronellate & -4.6 & 171 & (E)- $\beta$-ocimene & -4.5 \\
\hline 46 & $\delta$-Terpineol & -5.2 & 109 & Exo-2-hydroxycineole acetate & -5.2 & 172 & E-linalool oxide & -5.0 \\
\hline 47 & p-Mentha-1 (7),8-dien-2-ol & -5.0 & 110 & (E)-Methyl isoeugenol & -5.0 & 173 & p-cymenene & -4.5 \\
\hline 48 & p-Cymen-8-ol & -5.0 & 111 & $\gamma$-Elemene & -4.9 & 174 & $\begin{array}{c}\text { E-sabinene hydrate } \\
\text { acetate }\end{array}$ & -4.9 \\
\hline 49 & Trans-Carveol & -4.7 & 112 & Cubenol & -5.4 & 175 & E-Thujone & -4.6 \\
\hline 50 & Piperitol & -7.7 & 113 & Thuja-2,4(10)-diene & -4.5 & 176 & Nerol & -4.8 \\
\hline 51 & Epi-Globulol & -5.3 & 114 & Terpinolene & -4.9 & 177 & Z-carveol & -4.6 \\
\hline 52 & Globulol & -5.6 & 115 & Nopinone & -4.5 & 178 & Cumin aldehyde & -4.8 \\
\hline 53 & $\alpha$-Eudesmol & -5.6 & 116 & Sabina ketone & -4.5 & 179 & E-sabinyl acetate & -5.1 \\
\hline 54 & Sabinene & -4.4 & 117 & Carvacrol acetate & -5.4 & 180 & E- $\beta$-ionone & -5.2 \\
\hline 55 & Phenylethyl alcohol & -4.3 & 118 & $\alpha$-elemene & -5.6 & 181 & E-Longipinanol & -5.5 \\
\hline 56 & Indole & -4.4 & 119 & $\beta$-Copaene & -5.4 & 182 & Mustakone & -5.8 \\
\hline 57 & $\beta$-bourbonene & -5.3 & 120 & $\alpha$-Guaiene & -5.7 & 183 & Elemol acetate & -5.4 \\
\hline 58 & $\beta$-elemene & -4.9 & 121 & Trans- $\beta$-Elemenone & -5.4 & 184 & Thujopsenal & -5.4 \\
\hline 59 & $\alpha$-humulene & -5.7 & 122 & $\alpha$-phellandrene & -4.7 & 185 & Mintsulfide & -5.2 \\
\hline 60 & Trans- $\beta$-Farnesene & -5.2 & 123 & $(Z)$ - $\beta$-ocimene & -4.1 & 186 & $\beta$-eudesmol acetate & -5.9 \\
\hline 61 & $\beta$-Ionone & -5.2 & 124 & Allo-ocimene & -4.4 & 187 & $\begin{array}{l}\text { 5E,9E-Farnesyl } \\
\text { Acetone }\end{array}$ & -4.5 \\
\hline 62 & Germacrene D & -5.5 & 125 & Carvacrol & -4.9 & & Chloroquine & -5.9 \\
\hline 63 & Pentadecane & -4.4 & 126 & (Z)-Nerolidol & -5.2 & & Hydroxychloroquine & -6.6 \\
\hline
\end{tabular}

The best energies of interaction with SARS-CoV-2 main protease are observed for Piperitol, Warfarin, Abietatriene, cis-calamenen-10-ol, $\alpha$-Cadinene and Retinol (Table 3); so, these compounds could have more inhibitory potential against SARS-CoV-2 main protease than the other studied compounds. 
Table 3. Structures with the best Affinity in the binding pocket of SARS-CoV-2 main protease.

\begin{tabular}{|c|c|c|c|c|c|}
\hline $\mathbf{N}^{\circ}$ & $\begin{array}{c}\text { Name of } \\
\text { molecules }\end{array}$ & Plant of origin & $\begin{array}{c}\text { Affinity } \\
\text { (Kcal/mol) }\end{array}$ & Structure 2D & Structure 3D \\
\hline 50 & Piperitol & Eucalyptus sp. & -7.7 & & \\
\hline 105 & Warfarin & Glycyrrhiza glabra & -7.1 & & \\
\hline 73 & Abietatriene & Juniperusoxycedrus $L$. & -6.4 & & \\
\hline 167 & $\begin{array}{l}\text { Cis- } \\
\text { calamenen- } \\
10 \text {-ol }\end{array}$ & Thymus munbyanus & -6.3 & & \\
\hline 64 & $\alpha$-Cadinene & $\begin{array}{l}\text { Marrubium vulgare L, } \\
\text { Thymus numidicus Poiret and } \\
\text { Teucrium polium }\end{array}$ & -6.1 & & \\
\hline 104 & Retinol & Glycyrrhiza glabra & -6.1 & & \\
\hline
\end{tabular}

The result of interactions between Piperitol and the studied enzyme (Figure 1) shows Conventional Hydrogen Bond with Gly 143 and Ser 144 residues, Carbon Hydrogen bonding with Phe 140 and Gln 189 residues and $\pi$-Alkyl with Cys 145, His 172 and His 163 residue. 


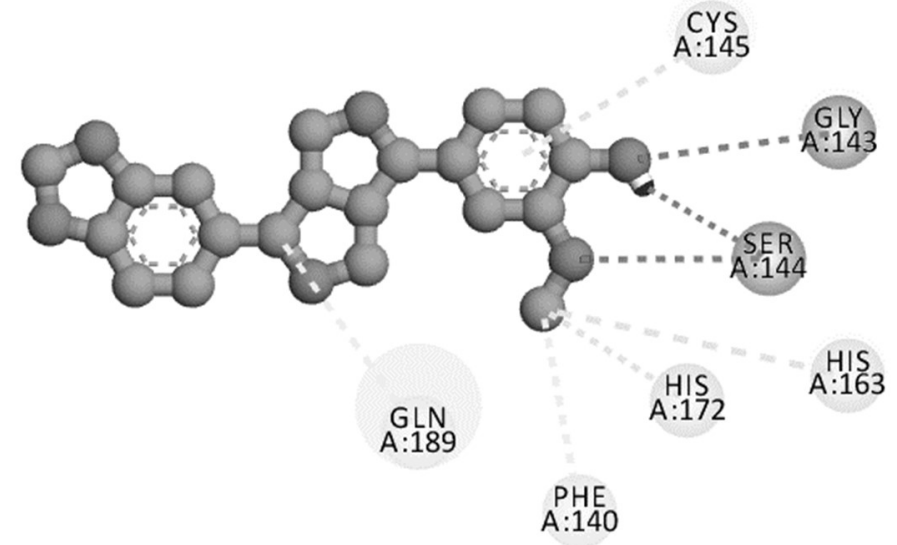

Interactions
Conventional Hydrogen Bond
$\square$ Carbon Hydrogen Bond

Pi-Alkyl

Figure 1: Interactions between Piperitol and SARS-CoV-2 main protease.

The presence of hydrogen bonding in the complex formed by Piperitol and the studied enzyme increase the affinity of the complex and gives to Piperitol a pharmacological importance; actually hydrogen bonds play a major role in the pharmacological effect of ligands [22, 61].

The result of interactions between Warfarin and the studied enzyme (Figure 2) shows Conventional Hydrogen Bond with Glu 166, Ser 144 and Cys 145 residues, $\pi-\pi$ T-shaped with His 41 residue and $\pi$-Alkyl with Met 164 residue.

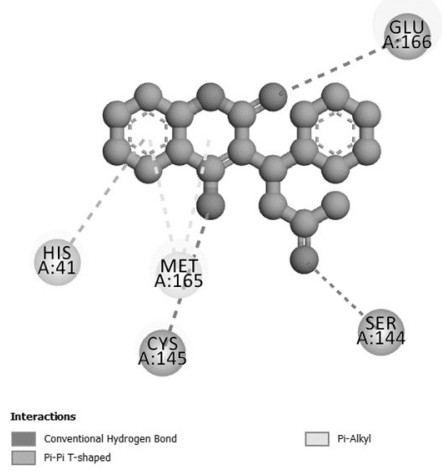

Figure 2: Interactions between Warfarin and SARS-CoV-2 main protease.

The affinity of Piperitol, Warfarin, Abietatriene, cis-calamenen-10-ol, $\alpha$-Cadinene and Retinol in the binding pocket of the studied enzyme can be explained by the number and type of bonds noticed in formed complexes, indeed the presence of hydrogen bonds shows an important potential pharmacological effect, by the inhibition of SARS-CoV-2 main protease. The inhibition of this protein will induce the inhibition of viral replication; these results show that these molecules could be interesting in the clinical management of COVID-19.

\subsection{Lipinski's Rule and ADMET Prediction}

The Lipinski's rule and ADMET parameters of the selected compounds, the inhibitor (N3), chloroquine and hydroxychloroquine were calculated using pkCSM and Swissadmet web servers. The Lipinski's rule including $\log P$, number of hydrogen bonds acceptor, number hydrogen bonds donor, number of rotatable bonds and molecular weight were shown in Table 4.

Table 4. Lipinski's rule of potential inhibitors, N3, chloroquine and hydroxychloroquine.

\begin{tabular}{|c|c|c|c|c|c|c|}
\hline \multirow{2}{*}{ Compounds } & \multicolumn{5}{|c|}{ Property } & \multirow{2}{*}{$\begin{array}{c}\text { Lipinski } \\
\text { violations }\end{array}$} \\
\hline & $\log P$ & H-bond Acceptor & H-bond Donor & Rotatable bonds & Molecular weight g/mol & \\
\hline Rule & $<4.15$ & $\leq 10$ & $<5$ & $<10$ & $\leq 500$ & $\leq 1$ \\
\hline Piperitol & 1.57 & 6 & 1 & 3 & 356.37 & 0 \\
\hline Warfarin & 2.51 & 4 & 1 & 4 & 308.33 & 0 \\
\hline Abietatriene & 6.64 & 0 & 0 & 1 & 270.45 & 1 \\
\hline Cis-calamenen-10-ol & 3.46 & 1 & 1 & 1 & 218.33 & 0 \\
\hline$\alpha$-Cadinene & 4.63 & 0 & 0 & 1 & 204.35 & 1 \\
\hline Retinol & 4.48 & 1 & 1 & 5 & 286.45 & 1 \\
\hline N3 & 0.61 & 9 & 6 & 23 & 680.79 & 3 \\
\hline Chloroquine & 3.20 & 2 & 1 & 8 & 319.87 & 0 \\
\hline Hydroxychloroquine & 2.35 & 3 & 2 & 9 & 335.87 & 0 \\
\hline
\end{tabular}

All the selected compounds respect the conditions mentioned in Lipinski's rule. The co-crystallized ligand presented three Lipinski Violations, whereas chloroquine and hydroxychloroquine respect all conditions of Lipinski's rule. The ADMET prediction was used in this work to calculate the pharmacokinetics parameters of selected compounds (Table 5).

Table 5. In silico ADMET prediction and synthetic accessibility values of selected compounds, chloroquine and hydroxychloroquine.

\begin{tabular}{|c|c|c|c|c|c|c|c|c|}
\hline \multirow{6}{*}{ Comp. } & \multicolumn{2}{|c|}{ Absorption } & \multicolumn{2}{|c|}{ Distribution } & \multirow{2}{*}{$\begin{array}{c}\text { Metabolism } \\
\text { CYP450 }\end{array}$} & \multicolumn{2}{|r|}{ Excretion } & \multirow{2}{*}{$\begin{array}{l}\text { Synthetic } \\
\text { accessibility }\end{array}$} \\
\hline & $\begin{array}{c}\text { Water } \\
\text { solubility }\end{array}$ & $\begin{array}{c}\text { Intestinal absorption } \\
\text { (human) }\end{array}$ & \begin{tabular}{|c|} 
Volume of \\
distribution
\end{tabular} & $\begin{array}{c}\text { Blood-brain barrier } \\
\text { Permeability CYP }\end{array}$ & & \multirow{2}{*}{\begin{tabular}{|c|}
$\begin{array}{c}\text { Total } \\
\text { clearance }\end{array}$ \\
3A4
\end{tabular}} & \multirow[t]{2}{*}{\begin{tabular}{|l|} 
hERG I inhibitor - \\
hERG II inhibitor
\end{tabular}} & \\
\hline & & & & & \begin{tabular}{|l|l|l|l|l|l|} 
2D6 & $3 \mathrm{~A} 4$ & $1 \mathrm{AA2}$ & $2 \mathrm{C} 19$ & $2 \mathrm{C} 9$ & $2 \mathrm{D} 6$ \\
\end{tabular} & & & \\
\hline & & & & & Inhibition & & & \\
\hline & & Num & & & Categorical & Numeric & Categorical & \\
\hline & $(\log \mathrm{mol} / \mathrm{l})$ & (\% absorbed) & $(\log \mathrm{L} / \mathbf{K g})$ & $(\log B B)$ & (yes/No) & \begin{tabular}{|l}
$(\log \mathrm{ml} /$ \\
$\mathrm{min} / \mathrm{Kg})$
\end{tabular} & (yes/ No) & Numeric \\
\hline Piperitol & -3.975 & 97.647 & -0.314 & -0.265 & No Yes Yes Yes Yes No Yes & 0.053 & No-No & 4.19 \\
\hline Warfarin & -4.513 & 96.161 & -0.266 & -0.17 & No Yes Yes Yes Yes No Yes & 0.719 & No-No & 3.79 \\
\hline Abietatriene & -6.749 & 95.083 & 1.54 & 0.628 & No Yes Yes Yes No No No & 0.961 & No-Yes & 3.18 \\
\hline Cis-calamenen-10-ol & -4.13 & 92.95 & 0.868 & 0.215 & No Yes Yes No No No No & 1.161 & No-No & 2.84 \\
\hline$\alpha$-Cadinene & -5.851 & 97.173 & 0.695 & 0.791 & No No $^{\text {No }}$ No No No & 1.18 & No-No & 4.35 \\
\hline Retinol & -6.404 & 91.728 & 0.497 & 0.642 & No Yes Yes No No No No & 1.531 & No-Yes & 4.28 \\
\hline Chloroquine & -4.249 & 89.950 & 1.332 & 0.349 & Yes Yes No No No Yes No & 1.092 & No-Yes & 2.76 \\
\hline Hydroxychloroquine & -3.627 & 90.217 & 1.076 & 0.074 & Yes Yes Yes No No Yes No & 1.152 & No-Yes & 2.82 \\
\hline
\end{tabular}


The results of ADMET analysis show that all the selected molecules have a low values for brain blood partition coefficient, were found indicating that they will have a very low potential to cross the brain-blood brain barrier thereby eliminating the possibility of CNS related toxicity. Additionally, the other pharmacokinetics parameters such as human intestinal absorption (HIA), water solubility $(\log \mathrm{mol} / \mathrm{L})$, metabolism and Synthetic Accessibility are all acceptable. The toxicity indicated by predicted hERG I and hERG II inhibitors shows that all selected compounds show hERG inhibition properties except Abietatriene and Retinol, that present hERG II inhibition, as well as for chloroquine and hydroxychloroquine.

Piperitol, Warfarin, cis-calamenen-10-ol and $\alpha$-Cadinene are the structures with best affinity in the binding site of the enzyme and all of them respect the conditions mentioned in Lipinski's rule and have acceptable ADMET proprieties; so, these compounds could have more potent antiviral treatment of COVID-19 than the studied compounds, and they have important pharmacokinetic properties and bioavailability than chloroquine and hydroxychloroquine.

\section{CONCLUSION}

In this study, we have tried to carry out a docking study of 187 natural compounds in the active site of SARS-Cov-2 main protease. The result indicates that Piperitol, Warfarin, cis-calamenen-10-ol, and $\alpha$-Cadinene are the structures with best affinity in the binding site of the enzyme and all of them respect the conditions mentioned in Lipinski's rule and have acceptable ADMET proprieties; so, these compounds could have more potent antiviral treatment of COVID-19 than the studied compounds, and they have important pharmacokinetic properties and bioavailability than chloroquine and hydroxychloroquine. The synthesis of these molecules and the evaluation of their in vitro activity against COVID-19 could be interesting.

\section{CONFLICT OF INTEREST}

No potential conflict of interest was reported by the author(s).

\section{REFERENCES}

1. Zhong, N., Zheng, B., Li, Y. et al. Epidemiology and cause of severe acute respiratory syndrome (SARS) in Guangdong, People's Republic of China, in February, 2003. The Lancet (2003);362(9393):1353-1358.

2. Wang, N., Shi, X., Jiang, L. et al. Structure of MERS-CoV spike receptorbinding domain complexed with human receptor DPP4. Cell Res. (2013); 23(8):986-993.

3. Kaul, D. An overview of coronaviruses including the SARS-2 coronavirusMolecular biology, epidemiology and clinical implications. Curr. Med. Res. Pract. (2020);10: 54-64.

4. Balkhair, A.A. COVID-19 Pandemic: A New Chapter in the History of Infectious Diseases. Oman Med. J. (2020); Vol. 35, No. 2: e123.

5. Gorbalenya, A. E., Baker, S.C., Baric, R.S. et al. Severe acute respiratory syndrome-related coronavirus - the species and its viruses, a statement of the Coronavirus Study Group. Preprint at bioRxiv. (2020).

6. Peng, M. Outbreak of COVID-19: An emerging global pandemic threat. Biomed. Pharmacother. (2020) ;129: 110499,

7. Wu, D., Wu, T., Liu, Q., et al. The SARS-CoV-2 outbreak: What we know. Int. J. Infecect. Dis. (2020); 94:44-48.

8. Chauhan, S. Comprehensive review of coronavirus disease 2019 (COVID19). Biomed. J. (2020); 43(4): 334-340.

9. Juan A. Siordia Jr., J.A. Epidemiology and clinical features of COVID-19: A review of current literature. J. Clin Virol. (2020); 127: 104357.

10. Sohrabi, C., Alsafi, Z., O'Neill N. et al. World Health Organization declares global emergency: A review of the 2019 novel coronavirus (COVID-19). Int. J. Surg. (2020); 76: 71-76.

11. Lee D.Y.W, Li, Q.Y., Liu, J., Efferth T. Traditional Chinese herbal medicine at the forefront battle against COVID-19: Clinical experience and scientific basis. Phytomed. (2020).

12. Palacios Cruz, M., Santos E., Velázquez Cervantes, M.A. COVID-19, a worldwide public health emergency. Rev. Clin. Esp. (2020).

13. Kwon, S., Lee, W, Jin, C., Jang, I., Jung, W-S., Moon, S-K., Cho, S-K. Could herbal medicine (Soshihotang) be a new treatment option for COVID-19? a narrative review. Integr. Med. Res. (2020); 9(3), 100480.

14. Huang, F., Li, Y., Leung, E. L-H. et al. A review of therapeutic agents and Chinese herbal medicines against SARSCOV-2 (COVID-19). Pharmacol. Res. (2020) 158, 1049292.
15. Leung, E.-L. H., Pan, H. D., Huang, Y. F. et al., The Scientific Foundation of Chinese Herbal Medicine against COVID-19, Eng.

16. Luo, L., Jiang, J., Wang, C. et al. Analysis on herbal medicines utilized for treatment of COVID-19. Acta Pharm. Sin. B (2020); 10(17): 1192-1204.

17. Ang, L., Lee, H.W., Choi, J.Y. et al. Herbal medicine and pattern identification for treating COVID-19: a rapid review of guidelines. Integr. Med. Res. (2020); 9: 100407.

18. Ang, L., Lee, H.W., Kim A. et al. Herbal medicine for the management of COVID-19 during the medical observation period: A review of guidelines. Integr. Med. Res. (2020); 9: 100465.

19. Panyod, S., Ho, C-T., Sheen, L-Y. Dietary therapy and herbal medicine for COVID-19 prevention: A review and perspective. J. Trad. Compl. Med. (2020) ; 10(4): 420-427

20. Baildya, N., Ghosh, N.N., Chattopadhyay, A.P. Inhibitory activity of hydroxychloroquine on COVID-19 main protease: An insight from MDsimulation studies. J. Mol. Struct. (2020); 1219:128595.

21. Marmor, M.F., Kellner, U., Lai, T.Y., et al. Recommendations on screening for chloroquine and hydroxychloroquine retinopathy (2016 revision). Ophthalmol. (2016); 123:1386-1394.

22. Xian, Y., Zhang, J., Bian, Z. et al. Bioactive natural compounds against human coronaviruses: a review and perspective. Acta Pharm. Sin. B (2020); 10(7):1163-1174

23. McKeea, D.L., Sternberg, A., Stange, U. et al. Candidate drugs against SARS-CoV-2 and COVID-19. Pharmacol. Res. (2020) ;157: 104859.

24. Kouznetsov, V.V. COVID-19 treatment: Much research and testing, but far, few magic bullets against SARS-CoV-2 coronavirus. Eur. J. Med. Chem. (2020); 203: 112647 .

25. Abderrahim, A., Belhamel, K., Chalchat, J-C., et al. Chemical composition of the essential oil from Artemisia arborescens L. growing wild in Algeria Rec Nat Prod. (2010); 4:87-90.

26. Apostolico, I., Aliberti, L., Caputo, L., et al. Chemical composition, antibacterial and phytotoxic activities of Peganum harmala seed essential oils from five different localities in Northern Africa. Molecules. (2016); 21:1235.

27. Dob, T., Berramdane, T., Chelgoum, C. Chemical composition of essential oil of Pinus halepensis Miller growing in Algeria. Comptes Rendus Chim. (2005); 8:1939-1945.

28. Ourzeddine, W., Fadel, H., Mechehoud, Y., et al. Chemical Composition and Antioxidant Activity of the Fruit Essential Oil of Zizyphus lotus (L.) Desf. (Rhamnaceae). (2017); 9(2):228-232.

29. Laouer, H., Yabrir, B., Djeridane, A., et al. Composition, antioxidant and antimicrobial activities of the essential oil of Marrubium deserti. Nat Prod Commun. (2009); 4(8):1133-1138.

30. Benayache, S., Benayache, F., Benyahia, S., et al. Leaf Oils of some Eucalyptus Species Growing in Algeria. J Essent Oil Res. (2001); 13:210213.

31. Yabrir, B. Essential Oil of Marrubium vulgare: Chemical Composition and Biological Activities. A Review. Nat Prod Sci. (2019); 25:81-91.

32. Dob, T., Dahmane, D., Chelghoum, C. Essential Oil Composition of Juniperus Oxycedrus. Growing in Algeria. Pharm Biol. (2006); 44:1-6.

33. Benabdallah, F.Z., Kouamé, R.O., El Bentchikou, M., et al. Études ethnobotanique, phytochimique et valorisation de l'activité antimicrobienne des feuilles et de l'oléorésine du pistachier de l'atlas (Pistacia atlantica Desf.) Phytothérapie. (2017); 15:222-229.

34. Lograda, T., Chaker, A.N., Chalard, P., et al. Chemical composition and antimicrobial activity of essential oil of Genista numidica Spach. and G. saharae Coss et Dur. Asian J Plant Sci. (2009); 8:495-499.

35. Boutaghane, N., Kabouche, A., Touzani, R., et al. GC/MS Analysis and Analgesic Effect of the Essential Oil of Matricaria pubescens from Algeria. Nat Prod Commun. (2011); 6(2):1251-252.

36. Halla, N., Heleno, S.A., Costa, P., et al. Chemical profile and bioactive properties of the essential oil isolated from Ammodaucus leucotrichus fruits growing in Sahara and its evaluation as a cosmeceutical ingredient. Ind Crops Prod. (2018); 119:249-254

37. Chouitah, O. The essential oil of Algerian Ephedra alata subsp. alenda and its antimicrobial properties. J New Biol Rep. (2019); 8(3): 190-193.

38. Chouitah, O. Chemical Composition and Antimicrobial Activities of the Essential Oil from Glycyrrhiza glabra Leaves. J Essent Oil-Bear Plants. (2011); 14(3):284-288.

39. Berka-Zougali, B., Ferhat, M-A., Hassani, A., et al. Comparative Study of Essential Oils Extracted from Algerian Myrtus communis L. Leaves Using Microwaves and Hydrodistillation. Int J Mol Sci. (2012); 13:4673-95.

40. Achoub, H., Zaiter, L., Benayache, F., et al. Chemical Composition of the Essential Oil of Aerial Parts of Thymus ciliatus (Desf.). Acta Sci Nat. (2019); $6: 62-70$. 
41. Benabed, H.K., Gourine, N., Ouinten, M., et al. Chemical Composition, Antioxidant and Antimicrobial Activities of the Essential Oils of Three Algerian Lamiaceae Species. Curr Nutr Food Sci. (2017); 13:97-109.

42. Messara, Y., Fernane, F., Meddour, R. Chemical composition, antibacterial, and antifungal activities of the essential oil of Thymus numidicus Poiret from Algeria. Phytothérapie (2018); 16(3): 163-168.

43. Hadj Khelifa, L., Brada, M., Brahmi, F., et al. Chemical composition and antioxidant activity of essential oil of Ocimum basilicum leaves from the northern region of Algeria. J Herb Med. (2012); 1:53-58.

44. Brada, M., Khelifa, L.H., Achour, D., et al. Essential oil composition of Ocimum basilicum L. and Ocimum gratissimum L. from Algeria. J Essent Oil Bear Plants. (2011); 14:810-814.

45. Bendif, H., Boudjeniba, M., Miara, M.D., et al. Essential Oil of Thymus munbyanus subsp. coloratus from Algeria: Chemotypification and in vitro Biological Activities. Chem Biodivers. (2017) ; 14(3).

46. Bendif, H. Caractérisation phytochimique et détermination des activités biologiques in vitro des extraits actifs de quelques Lamiaceae: Ajuga iva (L.) Schreb., Teucrium polium L., Thymus munbyanus subsp. coloratus (Boiss. \&amp; Reut.) Greuter \&amp; Burdet et Rosmarinus eriocalyx Jord \&amp. [dissertation]. ENS Kouba Algeria; (2017).

47. Quezel, P., Santa, S. Nouvelle Flore de l'Algérie et des régions désertiques méridionales. Paris : CNRS ; (1963).

48. IUCN International Union for Conservation of Nature. A guide to medicinal plants in north Africa. Malaga, Spain: IUCN Center for Mediterranean Cooperation; (2005).

49. Pence, H.E., Williams, A. ChemSpider: An Online Chemical Information Resource. J Chem Educ. (2010); 87:1123-1224.

50. Protein Data Bank PDB. http://www.rcsb.org.
51. Trott, O., Olson, A.J. AutoDock Vina: improving the speed and accuracy of docking with a new scoring function, efficient optimization, and multithreading. J Comput Chem. (2010); 31:455-61.

52. Hunter, C.A., Lawson, K.R., Perkins, J., Urch, C.J. Aromatic interactions. J Chem Soc Perkin Trans 2. (2001); 0:651-669.

53. Melissa, R. Pitman and Ian Menz, R. in Applied Mycology and Biotechnology, D. K. Arora, R. M. Berka, G. B. Singh eds. Elsevier B. V. Netherlands, 2006; pp.37-59.

54. Dassault Systèmes BIOVIA Discovery Studio Modeling Environment. Release 2017 Dassault Systèmes (2016).

55. Belhassan, A., Zaki, H., Aouidate, A., et al. Interactions between (4Z)-hex4-en-1-ol and 2-methylbutyl 2-methylbutanoate with olfactory receptors using computational methods. Mor J Chem. (2019); 7(1):028-035.

56. Hakmi, M., Bouricha, E.M., Kandoussi, I., et al. Repurposing of known antivirals as potential inhibitors for SARS-CoV-2 main protease using molecular docking analysis. Bioinformation. (2020); 16(4):301-306.

57. Jalkute, C.B., Barage, S.H., Dhanavade, M.J.. Identification of Angiotensin Converting Enzyme Inhibitor: An in Silico Perspective. Int J Pept Res Ther. (2015); 21: 107-115.

58. Pires, D.E.V., Blundell, T.L., Ascher, D.B.. pkCSM: Predicting SmallMolecule Pharmacokinetic and Toxicity Properties Using Graph-Based Signatures. J Med Chem. (2015); 58(9):4066-4072.

59. Daina, A., Michielin, O., Zoete, V. Swiss ADME : a free web tool to evaluate pharmacokinetics, drug- likeness and medicinal chemistry friendliness of small molecules. Nat Publ Group. (2017); 1-13.

60. Zhao, Y.H., Abraham, M.H., Le, J., et al. Rate-Limited Steps of Human Oral Absorption and QSAR Studies. (2002); 19:1446-1457.

61. Belhassan, A., Chtita, S., Zaki, H., et al. Molecular docking analysis of Nsubstituted oseltamivir derivatives with the SARS-Cov-2 main protease. Bioinformation. (2020); 16:404-408. 